\title{
LOS COCCINÉLIDOS (COLEOPTERA: COCCINELLIDAE) DE LA REGIÓN DE MAGALLANES: NUEVOS REGISTROS Y DISTRIBUCIÓN REGIONAL
}

\author{
THE LADYBIRDS (COLEOPTERA: COCCINELLIDAE) FROM THE MAGELLAN \\ REGION: NEW RECORDS AND REGIONAL DISTRIBUTION
}

Álvaro Zúñiga-Reinoso ${ }^{1}$

\begin{abstract}
Although ladybirds are the most charismatic beetles, are practically unknown in the Magellan region. For this reason, the purpose of this study is to compile, update and submit new records of ladybirds, also provide regional distribution, biogeographical and ecological data. 13 species of ladybirds from Magellan region are recorded in the literature and five new records are added. We discussed their regional distribution and its possible relationship to phytogeographical regions proposed for Magellan region.
\end{abstract}

Key words: Ladybirds, Magallanes, new records, regional distribution, biogeography.

\section{RESUMEN}

A pesar de que las chinitas son un grupo muy carismático de coleópteros, son prácticamente desconocidos en la región de Magallanes. Es por esto, que el propósito de este trabajo es compilar, actualizar y entregar nuevos registros de las chinitas de la XII región, además de datos sobre su distribución regional, aspectos biogeográficos y datos ecológicos. En la literatura se registran 13 especies de coccinélidos para Magallanes y se adicionan cinco nuevos registros. Se discute sobre su distribución regional y su posible relación con las regiones fitogeográficas propuestas para Magallanes.

Palabras clave: Chinitas, Magallanes, nuevos registros, distribución regional, biogeografía.

1 Departamento de Zoología, Facultad de Ciencias Naturales y Oceanográficas, Universidad de Concepción, Casilla 160-C, Concepción. alvzuniga@udec.cl. 


\section{INTRODUCCIÓN}

Los Coccinellidae son una familia muy diversa y conocida dentro del orden Coleoptera. Se les conoce vulgarmente con el nombre de "chinitas" o "mariquitas", y debido a su inofensiva apariencia y sus vistosos colores son considerados como uno de los grupos de coleópteros más carismáticos. Dichas características fueron reconocidas desde temprano por el hombre, tomando características mágicoreligiosas (Zabala et al. 2003). Por otra parte, estos coleópteros son de gran interés para la agricultura, ya que tanto en su etapa adulta como larvaria son grandes depredadores de insectos herbívoros por lo que son utilizados para el control de importantes plagas agrícolas (Zúñiga 1967, 1985, Zúñiga et al. 1986).

A pesar de su potencial utilidad benéfica y de su proximidad con el hombre, estos coleópteros por muchos años tuvieron la atención de unos pocos especialistas en Chile. Los escasos trabajos son en su mayoría antiguos trabajos taxonómicos, referentes a la descripción de nuevas especies o revisiones de grupos (Brèthes 1925 a y b, Hoffman 1970, Gordon 1977, Gordon 1994), o relacionados con nuevos registros de coccinélidos para el país (Aguilera 1987, Aguilera \& Rojas 1990), o bien catálogos generales de coleópteros que sólo nominan a coccinélidos presentes en Chile (Elgueta \& Arriagada 1989, Arias 2000). Trabajos recientes y escasos tratan aspectos como la biología y ecología de estos insectos (Rebolledo et al. 2007). Sin embargo, hace aproximadamente cuatro años que se ha comenzado a reunir una gran cantidad de información a través de internet sobre la fauna de coccinélidos de Chile, tratando muchos aspectos como descripciones de nuevas especies, nuevos registros para nuestro país y algunos datos biológicos (González 2006¹ y colaboradores del sitio web: www.coccinellidae.cl).

De las aproximadamente seis mil especies descritas alrededor del mundo, tan solo 100 especies han sido citadas para el país, las que se encuentran distribuidas en cinco subfamilias: Exopelctrinae, Sticholotidinae, Scymnidae, Coccidulinae, Coccinellinae (González 2010²). Aún así, el número de especies podría seguir aumentando paulatinamente

1 González, G. 2006. Los Coccinellidae de Chile. www. coccinellidae.cl.

2 González, G. 2010. Lista actualizada de Coccinellidae de Chile. www.coccinellidae.cl. y ya se cuenta con mucho material que aún está sin describir (González 2006¹).

En la región de Magallanes, a pesar de ser más grande de Chile y con una gran variedad de ecotonos, se han descritos y citado unas pocas especies (González 2006¹, 2008, 2010²). Además, de las especies citadas para Magallanes, existen especies que tienen registros dudosos y cuyas presencias se deben confirmar para la región. La escasez de investigación, así como también el clima y la geografía de esta región, son algunos factores que influyen al bajo conocimiento existente sobre el tema, tratándose en la mayoría de los casos trabajos netamente taxonómicos (e.g Hoffman 1970, Gordon 1994), sin saber prácticamente nada sobre la biología, ecología y de la distribución de las especies de coccinélidos de esta región austral. Este trabajo tiene como propósito compilar y actualizar los datos preexistentes de los coccinélidos de Magallanes, entregando nuevos registros y aspectos referentes a la distribución regional y biogeografía de estos coleópteros.

\section{MATERIAL Y MÉTODOS}

Se revisó la literatura correspondiente a los Coccinellidae chilenos en busca de antecedentes, claves, ilustraciones, los que fueron usados para la identificación del material de Coccinellidae de Magallanes depositados en diferentes colecciones entomológicas. Las colecciones institucionales y colecciones particulares, además de muestras entomológicas que fueron revisadas son: Colección de Zoología del Instituto de la Patagonia, Universidad de Magallanes, Punta Arenas, Chile (CZIP); Museo Nacional de Historia Natural, Santiago, Chile (MNHN); Museo de Zoología, Universidad de Concepción, Concepción, Chile (UCCC); Colección de Entomología de la Universidad Metropolitana de Ciencias de la Educación, Santiago, Chile (UMCE); Muestra de Insectos del Parque Nacional Torres del Paine, Torres del Paine, Chile (MITP) y Colección Particular Álvaro Zúñiga, Punta Arenas, Chile (CPAZ) ${ }^{3}$.

Además se utilizaron sólo las localidades precisas de colecta registradas en colecciones y en la literatura para fabricar los mapas de distribución

3 La mayor parte del material de Coccinellidae de CPAZ, fue donado por el autor a la colección particular de Guillermo González. 
Tabla 1. Listado de especies y el elenco sistemático de los coccinélidos de la región de Magallanes en base a la revisión bibliográfica y de colecciones entomológicas. ${ }^{*}=$ Nuevos registros para la región de Magallanes. $+=$ Especies introducidas.

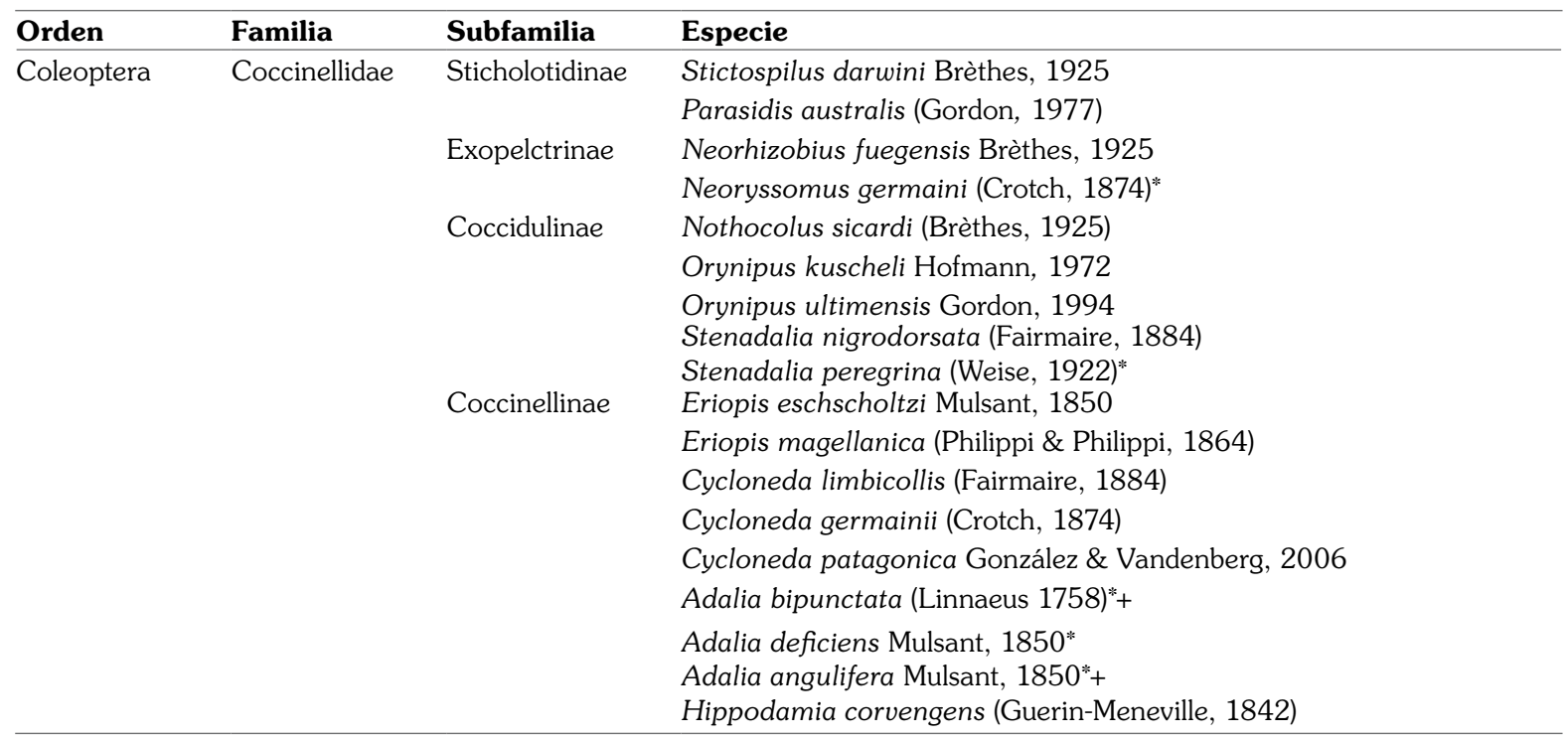

regional de las especies de coccinélidos de Magallanes. Como principal fuente de información bibliográfica y sistemática se trabajó en base a González (2006 2008 y $2010^{2}$ ).

\section{RESULTADOS}

En la literatura se registran 13 especies de chinitas para Magallanes, a las que se les adicionan cinco nuevos registros procedentes de las colecciones entomológicas revisadas. Los coccinélidos de Magallanes se encuentran distribuidos en 4 de las 5 subfamilias descritas para Chile (Tabla 1). Se detalla a continuación una diagnosis y las localidades de colecta regional para cada especie de Coccinellidae registrada en este estudio:

Eriopis eschscholtzi Mulsant, 1850 (Fig. 1:1)

De forma alargada y plana, color general negro. El pronoto y los élitros presentan manchas amarillas y anaranjadas. El pronoto presenta los bordes laterales y el borde anterior de color amarillo pálido, con una mancha en centro contigua al borde anterior y una mancha separada del borde en la base. Élitros negros con los bordes de color amarillo o anaranjado en la parte anterior, lateral y posterior, con dos manchas irregulares y contiguas al borde lateral y posterior, estas manchas forman dos franjas negras paralelas y perpendiculares al eje del cuerpo.

Esta especie es muy variable en su diseño elitral. Se han encontrado ejemplares en la región de la Araucanía con élitros completamente anaranjados y en la región de Aisén, ejemplares con las manchas anaranjadas laterales y posteriores separadas de los bordes. No se han registrado otras variedades en la región, salvo la que se describe. Según González (20061), la forma que se encuentra en Magallanes, correspondería a una forma cercana a maginicollis, las que serían más oscuras y parecidas a $E$. magellanica. Sin embargo el diseño del pronoto es un carácter estable e idóneo para identificar esta especie y diferenciarla de E. magellanica.

Esta especie se distribuye en Chile desde la Región de Valparaíso hasta la Región de Magallanes y la Antártica Chilena (González 2008). Localidad de colecta: Provincia de Última Esperanza: Parque Nacional Torres del Paine (CPAZ), Cerro Paine (Hoffman 1970). (Fig. 2:1).

Eriopis magellanica (Philippi \& Philippi, 1864) (Fig. 1:2)

Especie parecida a la anterior de forma alargada y plana, de color general negro. El pronoto y los 

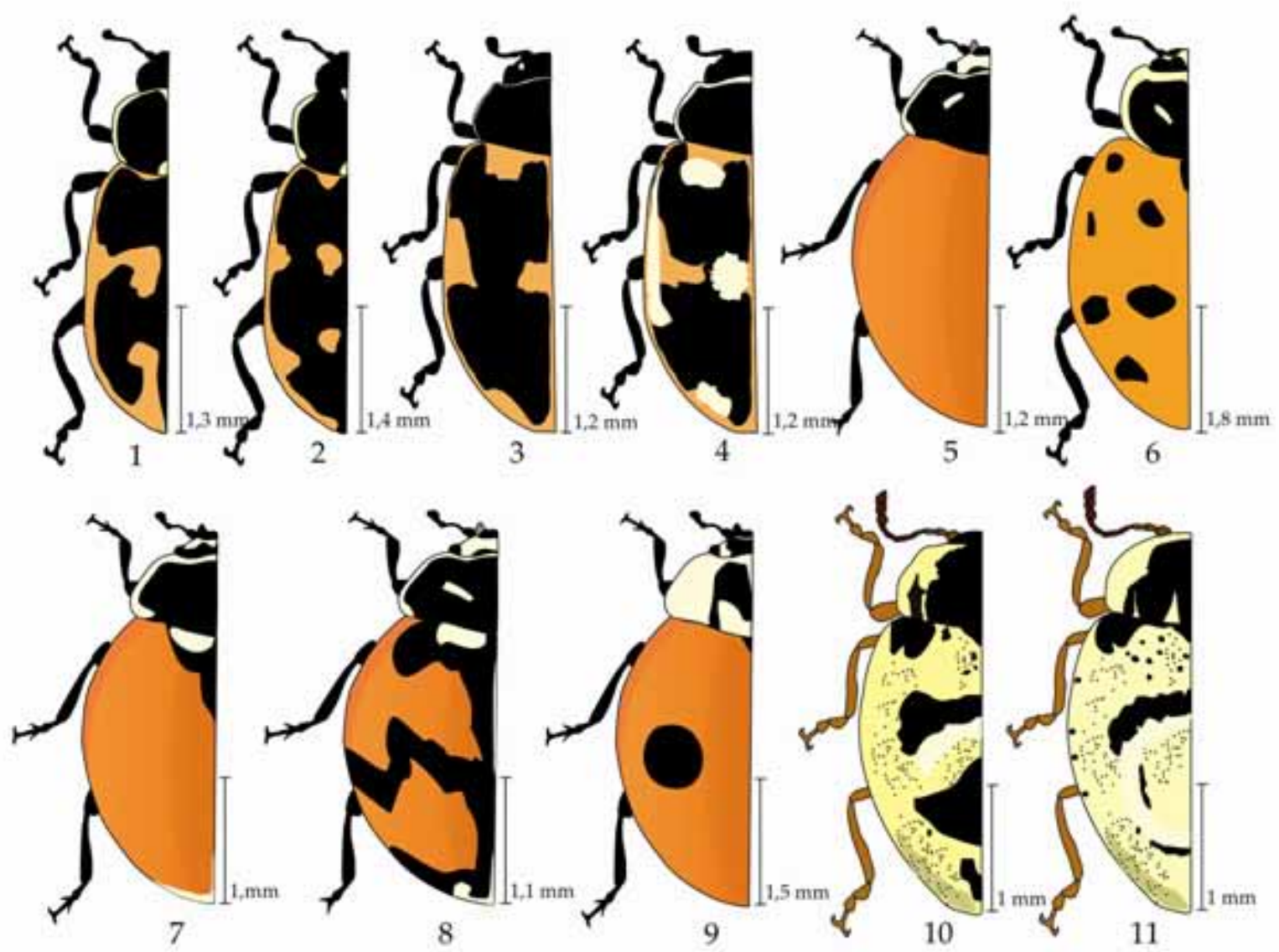

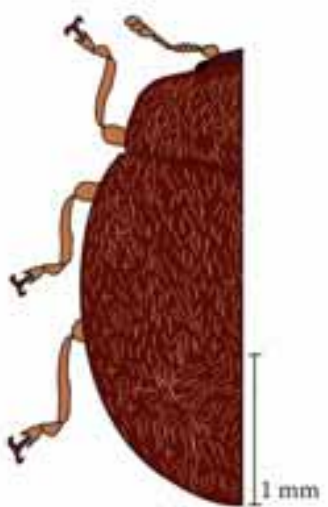

12

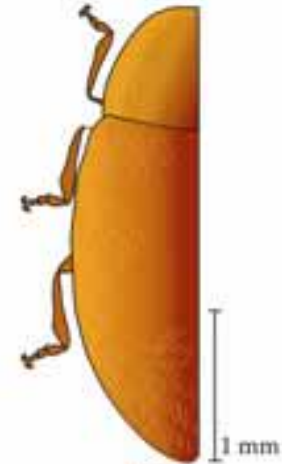

13

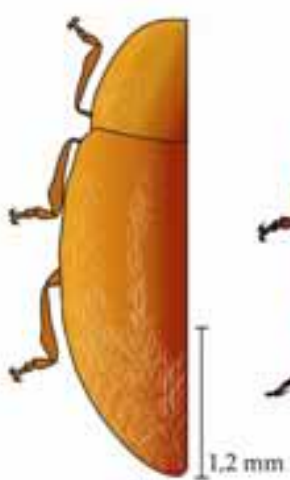

14

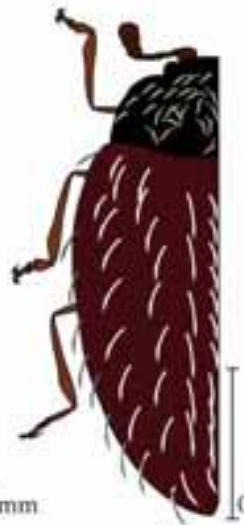

15

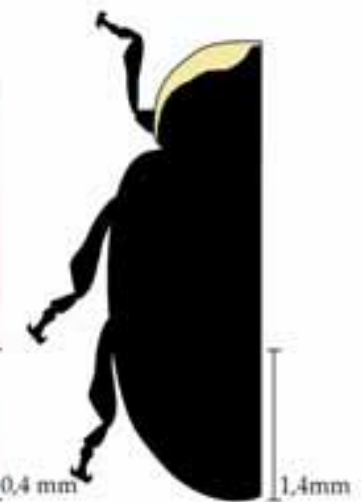

16

Fig. 1. Los coccinélidos de la región de Magallanes en vista dorsal del lado izquierdo: 1) Eriopis eschscholtzi, 2) Eriopis magellanica, 3) Cycloneda patagónica, 4) Cycloneda germainii, 5) Cycloneda limbicollis, 6) Hippodamia convergens, 7) Adalia deficiens, 8) Adalia angulifera, 9) Adalia bipunctata, 10) Stenadalia nigrodorsata, 11) Stenadalia peregrina, 12) Nothocolus sicardi, 13) Orynipus kuscheli, 14) Orynipus ultimensis, 15) Stictospilus darwini y 16) Neoryssomus germaini. Se excluyen de la figura las especies Parasidis australis y Neorhizobius fuegensis, ya que no se encontraron ejemplares de estas especies en las colecciones revisadas y son desconocidas por el autor. (C) Álvaro Zúñiga-Reinoso 


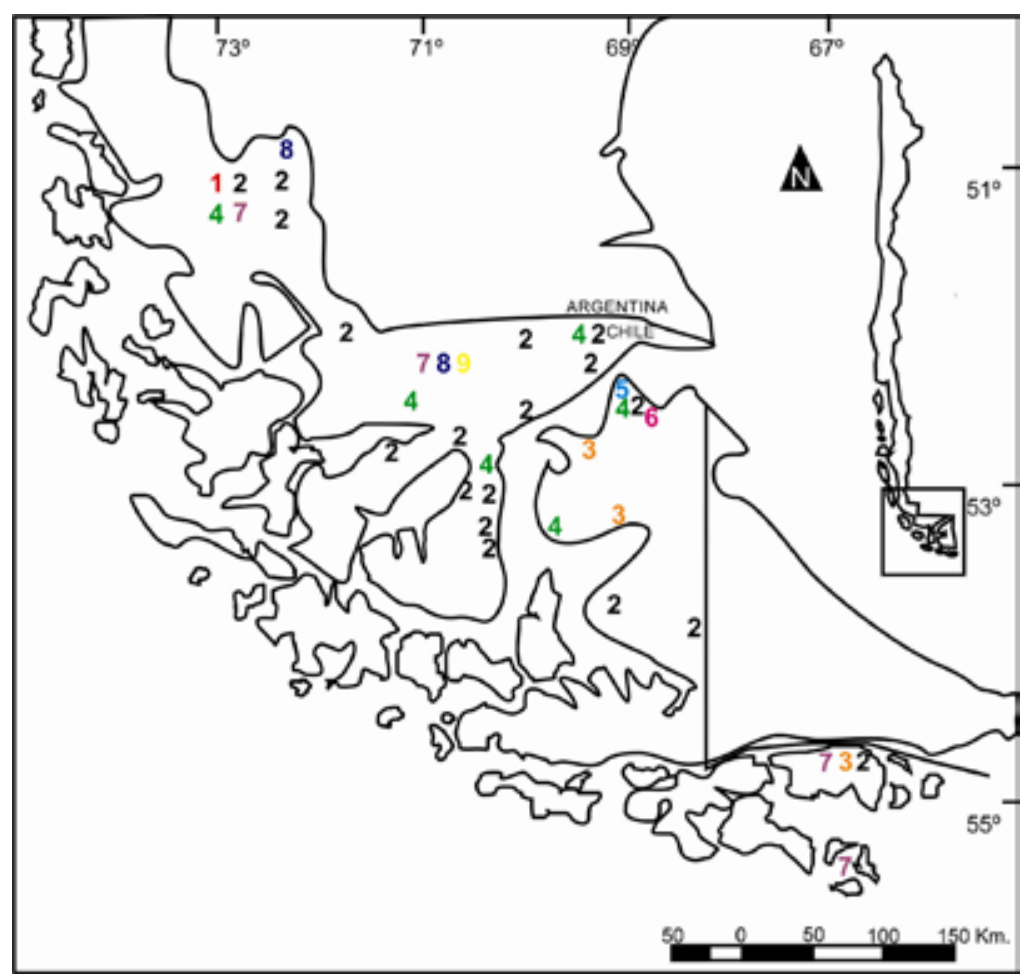

Fig. 2. Mapa de distribución regional de los Coccinélidos de Magallanes: 1) Eriopis eschscholtzi, 2) Eriopis magellanica, 3) Cycloneda patagónica, 4) Cycloneda germainii, 5) Cycloneda limbicollis, 6)

Hippodamia convergens, 7) Adalia deficiens, 8) Adalia angulifera, 9) Adalia bipunctata.

élitros presentan manchas amarillas y/o anaranjadas. El pronoto presenta bordes laterales de color amarillo pálido, además presenta dos manchas centrales; una mancha en el margen anterior y otra en la base del pronoto. Élitros negros con los bordes de color amarillos o anaranjados en la parte anterior, lateral y posterior, con 3 manchas irregulares y contiguas a los bordes, estas manchas forman 3 franjas negras paralelas y perpendiculares al eje del cuerpo. En el disco presenta dos manchas irregulares de color amarillas o anaranjadas, la primera cerca del ápice y la otra cerca de la base elitral.

Esta especie es la chinita más común de la región. Es muy variable en su diseño elitral, pudiendo encontrarse ejemplares en la región completamente negros, sin la mancha basal del disco o con las manchas elitrales anaranjadas muy desarrolladas. Sin embargo el diseño del pronoto es estable y puede ser usado como un buen carácter para diferenciar esta especie con la anterior.
Esta especie se distribuye en Chile en la zona austral del país, desde la región de Aisén hasta la región de Magallanes y Antártica Chilena (González 2008). Localidades de colecta: Provincia de Última Esperanza: Laguna Azul (MITP/UCCC), Rio Grey (CZIP), Lago Grey (CZIP), Laguna Amarga (CPAZ), Estancia Cerro Castillo (CZIP), Estancia Cerro Guido (UCCC), Morro Chico (Bajo Senecio patagonico) (CPAZ). Provincia de Magallanes: Parque Nacional Pali Aike (CZIP), Oezy Harbour (CZIP), Isla Riesco: Seno Skyring (CZIP), Seno Otway (UCCC), Mina Rica (UCCC), Punta Arenas (CPAZ/MNHN), Punta Arenas: Instituto de la Patagonia (CZIP/CPAZ), La Discordia (UMCE), Agua Fresca (UMCE), Leñadura (UCCC), Tres Puentes (Eduardo Faúndez com. pers.), Cabo Negro (UMCE), Punta Delgada (CZIP), Gallego Chico (Gonzalez 2006ㄹ). Provincia de Tierra del Fuego: Punta Espora (CZIP), Cerro Sombrero (CPAZ), Cameron (Gonzalez 2006¹), Vicuña (UM$\mathrm{CE})$. Comuna Cabo de Hornos y Antártica Chilena: Puerto Williams (UCCC). (Fig. 2:2). 
Cycloneda patagonica González \& Vandenberg, 2006 (Fig. 1:3)

De forma ovalada y semiconvexa, color general negro. Presenta pequeñas manchas de color claro delante de los ojos. El pronoto presenta una franja pálida a penas visible en los bordes laterales y en el borde anterior de color claro. Élitros negros con los bordes de color anaranjado en todos sus márgenes, presenta cuatro manchas irregulares contiguas los bordes en cada élitro, una en el centro del borde anterior y posterior, y una en el centro de cada borde lateral. Estas manchas anaranjadas forman una mancha negra con forma de $\mathrm{H}$ recostada en el disco elitral. Esta especie es muy parecida externamente a C. germainii. Ambas especies se pueden diferenciar debido a que el tercer antenómero de C. patagonica es muy corto y a penas más largo que el segundo antenómero (González \& Vandenberg, 2006).

Esta especie es conocida en Chile sólo para la región de Magallanes (González 2010²). Localidades de colecta: Provincia de Tierra del Fuego: Puerto Percy, Nose Peak, Bahía Inútil (González \& Vendenberg 2006). Comuna Cabo de Hornos, Puerto Williams (UCCC). (Fig. 2:3).

\section{Cycloneda germainii (Crotch, 1874) (Fig. 1:4)}

Especie muy similar a la anterior. En el pronoto presenta una franja clara visible en los bordes laterales y en el borde anterior. Élitros negros con los bordes de color anaranjado en todos sus márgenes, presenta tres manchas irregulares en cada élitro de color marfil hacia el disco y contiguas a los bordes anterior, posterior e interno de los élitros, una en el centro del borde anterior y posterior, y una en el centro del borde lateral de la sutura elitral. Además posee una mancha marfil alargada, que cubre gran parte en el borde externo de los élitros y contiguamente a esta mancha, aparece una mancha central e irregular semicuadrada de color anaranjado que se proyecta hacia el disco elitral. Estas manchas de color claro forman una mancha negra semejante a una letra " $H$ " recostada en el disco elitral.

La forma que se describe arriba es fácilmente distinguible de $C$. patagonica. Sin embargo existen patrones de diseño elitral en esta especie, el cual no posee las manchas marfiles y que sería una forma melanizada de C. germainii, conocida como forma "duplaris" (González \& Vendenberg 2006). La forma "duplaris" posee un diseño elitral muy parecido al de C. patagónica. Sin embargo, la característica de las antenas, es un buen carácter para distinguir entre ambas especies. La especie C. germainii posee antenas más alargadas, debido a que el tercer antenómero es más alargado que el segundo (González \& Vendenberg 2006).

Esta especie se distribuye en Chile desde la región de Coquimbo hasta la región de Magallanes y Antártica Chilena (González 2010²). Lugares de colecta: Provincia de Última Esperanza: Laguna Azul (CZIP), Lago Pehoe (CZIP). Provincia de Magallanes: Parque Nacional Pali Aike (CZIP), Cruce Fabres (CZIP), Cabeza del Mar (CPAZ). Provincia de Tierra del Fuego: Punta Espora (CZIP), Bahía Azul (bajo follaje de Senecio patagónico) (CPAZ), Porvenir (Guillermo González com. pers.). (Fig. 2:4).

\section{Cycloneda limbicollis (Fairmaire, 1884) (Fig. 1:5)}

De forma ovalada y convexa. La cabeza es color negra con una mancha marfil irregular, que puede cubrir la mayor parte de la cabeza o ser una pequeña mancha al costado de estos. Pronoto de color negro con una franja en los bordes laterales y el borde anterior de color marfil. Presenta en el pronoto dos rayas diagonales en forma de slash (/) a cada lado del disco. Élitros de color anaranjado, con los bordes algo más claros.

Especie que en Chile sólo ha sido registrada en las regiones Metropolitana, Maule y de Magallanes y Antártica Chilena (González 2010²). Localidad de colecta: Provincia de Tierra del Fuego: bahía Azul (bajo follaje de Senecio patagónico) (CPAZ). (Fig. 2:5).

\section{Hippodamia convergens (Guerin-}

Meneville, 1842) (Fig. 1:6)

De forma ovalada y plana. La cabeza es color negra con una mancha marfil irregular, que cubre el margen anterior y el centro de la cabeza. Pronoto de color negro con una franja en los bordes laterales y el borde anterior de color marfil. Presenta en el pronoto dos rayas diagonales en forma de "slash" ( $\backslash$ a cada lado del disco. Los élitros normalmente de color anaranjado, pudiendo algunas veces ser rojos, poseen 6 manchas irregulares negras en cada élitro. Estas manchas se distribuyen en cada 
élitro de la siguiente forma: tres manchas pequeñas equidistantes en la primera mitad elitral, dos cerca del margen lateral elitral y una en el disco elitral, y son equivalentemente a los puntos de intersección de un triángulo. Luego más abajo, en la segunda mitad elitral, se encuentran tres manchas negras de mayor tamaño que se distribuyen de la misma forma que las de más arriba. Por último, poseen una mancha negra semitriangular en el escutelo.

Esta especie es de origen neártico e introducida a Chile. Se distribuye en todo Chile (González 2010²). Localidad de colecta: Provincia de Tierra del Fuego: Punta Espora. (CZIP) (Fig. 2:6).

\section{Adalia deficiens Mulsant, 1850 (Fig. 1:7)}

De forma ovalada y convexa. La cabeza es color negra con una franja de color marfil que cubre todo el borde anterior. Pronoto de color negro con una franja gruesa de color marfil que cubre totalmente los bordes laterales y el borde anterior. Élitros de color anaranjado, con una mancha semicircular marfil en la base de cada élitro, cercana al escutelo $e$ inmediatamente debajo de esta, una gran mancha negra que ocupa los dos élitros, se asemeja a la letra griega psi $(\Psi)$, y su brazo posterior baja por la sutura elitral agudizándose hasta aproximadamente el primer cuarto de los élitros. En el ápice de ambos élitros, se encuentra una línea delgada de color marfil, que cubre el ápice y parte de los bordes del margen posterior y de la sutura elitral.

La forma que se describe arriba, es la única que se ha encontrado en la región de Magallanes, sin embargo, esta especie presenta una gran variedad de diseños en los élitros y en pronoto. En líneas generales, los otros diseños se parecen mucho a los de $A$. angulifera y a $A$. bipunctata. Sin embargo se diferencian debido a que $A$. angulifera posee dos manchas lineales en el pronoto y además en la mancha marfil apical que ambas especies poseen, A. angulifera posee una mancha circular al final de la mancha que va por el margen posterior. Por otra parte, A. deficiens presenta un morfo con dos mancha semiredondas en cada élitro, asemejándose a $A$. bipunctata, sin embargo esta última especie posee el pronoto casi completamente de color marfil y una mancha negra en el pronoto que se asemeja a una "M", característica que diferencia a ambas especies.
Esta especie se distribuye en Chile desde la región de Atacama hasta la región de Aisén (González 2010²). Localidades de colecta: Provincia de Ultima Esperanza: Baguales (UCCC). Provincia de Magallanes: Laguna Blanca (UCCC). (Fig. 2:7).

\section{Adalia angulifera Mulsant, 1850 (Fig. 1:8)}

De forma ovalada y convexa. La cabeza es color negra con una franja de color marfil que cubre todo el borde anterior. Pronoto de color negro con una franja gruesa de color marfil que cubre totalmente los bordes laterales y el borde anterior. Presenta en el pronoto dos rayas diagonales en forma de "slash" ( $($ ) a cada lado de la porción central del disco. Élitros de color anaranjado, que presenta una mancha semicircular marfil en la base cercana al escutelo y una línea del mismo color en el margen de la sutura elitral de cada élitro, que no alcanza a llegar a la base, ni al ápice de esta estructura. En la zona basal de casa élitro, una franja de color negro se proyecta de forma horizontal, hacia el borde lateral sin llegar a tocarlo, esta franja en su base se proyecta hacia abajo y a medida que desciende se va aguzando hasta la parte media de cada élitro, donde se vuelve a proyectar horizontalmente una franja zigzagueante y angulosa, que va hacia arriba, luego desciende, formando un ángulo muy agudo y luego en la última parte de su recorrido vuelve a descender hasta tocar el borde lateral. En la base de esta franja, se proyecta una franja que desciende hasta el ápice elitral, donde se ensancha justo antes de llegar al ápice y se extiende por borde posterior del élitro. En el ápice existe una pequeña mancha marfil que se expande linealmente por la sutura elitral y por el borde elitral posterior, en esta última zona y al final de esta mancha lineal marfil, se proyecta una mancha circular contigua del mismo color.

En algunos ejemplares, se pueden unir las franjas negras basales y centrales, justo cuando la franja central se proyecta hacia arriba y luego baja abruptamente. La punta que se forma puede proyectarse hasta alcanzar la franja basal, formando como consecuencia una mancha anaranjada semicircular en el disco elitral.

Esta especie se distribuye en Chile desde la región de Atacama hasta la región de Aysén (González 2010²). Localidades de colecta: Provincia de Última Esperanza: Parque Nacional Torres del Paine: 


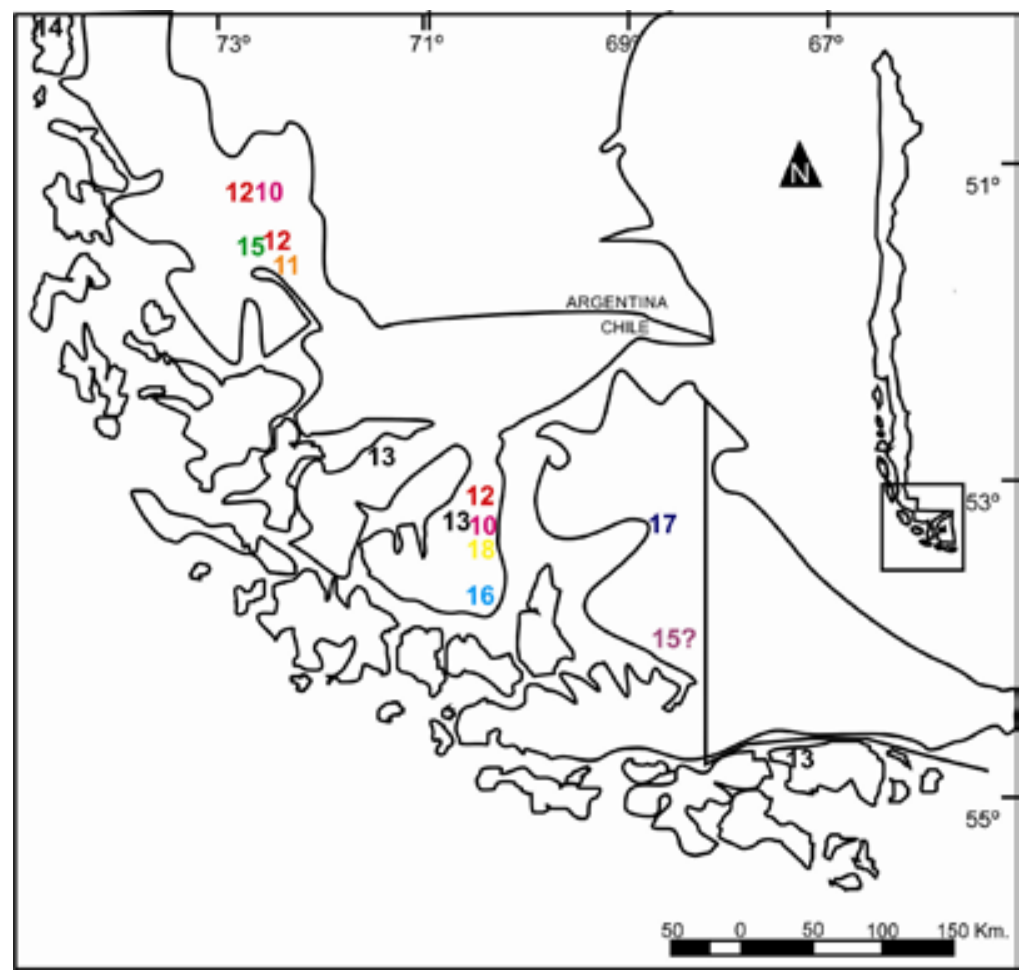

Fig. 3. Mapa de distribución regional de los Coccinélidos de Magallanes: 10) Stenadalia nigrodorsata, 11) Stenadalia peregrina, 12) Nothocolus sicardi, 13) Orynipus kuscheli, 14) Orynipus ultimensis, 15) Stictospilus darwini, 16) Neoryssomus germaini, 17) Neorhizobius fuegensis y 18) Parasidis australis.

Laguna Amarga (UCCC), Hostería las Torres (en follaje de Nothofagus antarctica) (CPAZ). Provincia de Magallanes: Laguna Blanca (UCCC). Comuna Cabo de Hornos: Puerto Williams, (UCCC). (Fig. 2:8).

\section{Adalia bipunctata (Linnaeus 1758) (Fig. 1:9)}

De forma ovalada y convexa. Cabeza de color negro, con pequeñas franjas de color marfil sobre los ojos. Pronoto de color marfil con una mancha negra en el disco con forma de letra "M". Élitros de color anaranjado, con una mancha circular en el disco en cada élitro.

Existen variaciones en el diseño y existen especímenes melánicos. Una de las variaciones se debe a que las manchas circulares de los élitros, se proyectan hacia los costados y en la parte central de la franja que se forma, la mancha también se proyecta hacia abajo. Las formas melánicas, son completamente negras con dos manchas anaranjadas en cada élitro, una semicuadrada en la base y una circular en el disco elitral o bien, la mancha en la base elitral, se transforma en una gran franja anaranjada y debajo de esta franja, las manchas circulares. Ninguna de estas variaciones se halló en este trabajo.

Esta especie es de origen paleártico e introducida a Chile. Se distribuye en el país desde la región de Tarapaca hasta la Araucanía, incluyendo Isla de Pascua (González 2010²).

Lugares de colecta: Provincia de Última Esperanza: Baguales (UCCC). Provincia de Magallanes: Laguna Blanca (UCCC). (Fig. 2:9).

\section{Stenadalia nigrodorsata}

(Fairmaire, 1884) (Fig. 1:10)

De forma elíptica y semiconvexa. De color general amarillo pálido e irregularmente jaspeado. Pronoto con una gran mancha negra que ocupa gran parte del disco, dejando un margen lateral irregular de color amarillo pálido que a veces se proyecta hacia el interior de esta mancha negra. Élitros amarillos pálidos con toques café claro, con 
manchas y puntos negro irregulares. En cada élitro existe una mancha irregular de color negro en la esquina que forman el borde lateral externo con el borde basal y en la esquina que forma el bode basal con la sutura elitral hay una o dos manchas irregulares, la que se disgregan hacia abajo y por la sutura en manchitas menores. En la parte central de los élitros se proyecta hacia los costados una franja irregular que no alcanza a tocar el borde, la cual en su parte distal se proyecta hacia abajo, agudizándose y abrazando una mancha que forma un arco horizontal de color blanquecino cuando los élitros están juntos. Debajo de este arco y en la segunda porción elitral se encuentra una gran mancha negra semitriangular que la base que nace en la sutura elitral y proyecta hacia los costados agudizándose sin tocar el borde lateral. Manchas irregulares negras se encuentran bajo esta última mancha y cercanos al ápice. Estas características son muy variables y poco constantes.

Esta especie se distribuye en Chile en la zona austral, desde la región de Aisén hasta la región de Magallanes y Antártica Chilena (González 2010²). Material examinado y citas: Provincia de Última Esperanza: Lago Pehoe (Gordon 1994), Torres del Paine (Guillermo González com. pers.). Provincia de Magallanes: Punta Arenas (Gordon 1994). (Fig. 3:10).

\section{Stenadalia peregrina (Weise, 1922) (Fig. 1:11)}

Especie muy similar a la anterior. De color general amarillo pálido e irregularmente jaspeado. Pronoto con una gran mancha negra que ocupa gran parte del disco, dejando un margen lateral irregular amplio de color amarillo pálido que a veces se proyecta hacia el interior de esta mancha negra. En la base del pronoto se proyectan hacia adelante dos manchas amarilla pequeñas a ambos lados del centro del pronoto, una triangular cercana al centro y una raya apenas visible más cercana al margen lateral. Élitros amarillos pálidos con toques café claro, con manchas y puntos negro irregulares. En cada élitro existe una mancha irregular de color negro en la esquina que forman el borde lateral externo con el borde basal que se une por una delgada franja que va por el borde basal con una mancha negra que se encuentra en el escutelo, esta última mancha disgrega hacia abajo y por la sutura en manchitas menores. En la parte central de los élitros se proyecta hacia los costados una franja irregular que no alcanza a tocar el borde, la cual se encuentra sobre una franja blanquecina que forma un circulo cuando los élitros están juntos. Dentro de este círculo, en la cara interna de la franja y en el punto más alejado de la sutura elitral, se encuentra una mancha negra irregular. Debajo de este círculo y en la segunda porción elitral se encuentra una pequeña franja negra horizontal que abraza por fuera la mancha circular. Manchas irregulares negra se encuentran bajo esta última mancha y cercanos al ápice.

El diseño corporal es muy variable y poco constante. Es muy fácil confundir a esta especie con S. nigrodorsata. Sin embargo, S. peregrina presenta pilosidad larga y densa en el pronoto que distingue a esta especie con la anterior (Gordon 1994).

Esta especie se distribuye en Chile desde la región de Valparaíso hasta la región de Aisén (González 2010²). Localidad de colecta: Provincia de Última Esperanza: Puerto Natales (UCCC). (Fig. 3:11).

\section{Nothocolus sicardi (Brèthes, 1925) (Fig. 1:12)}

De forma elíptica y semiconvexa, truncada en la porción anterior. Cuerpo completamente de color marrón rojizo o granate oscuro. En algunos casos, cabeza y pronoto más oscuro que el resto del cuerpo, de color marrón oscuro casi negro. Todo el cuerpo esta densamente cubierto de una pilosidad corta de color amarillo pálido.

Especie que se distribuye en Chile desde la región del Maule hasta la región de Magallanes y Antártica Chilena (González 2010²). Localidades de colecta: Provincia de Última Esperanza: Salto del Paine (Ex. Escalonia) (UCCC), Cruce Laguna Sofía (UCCC). Provincia de Magallanes: Punta Arenas, Parque Chabunco (Follaje de ñirre) (CPAZ), Seno Otway: Estancia Olga Teresa (UCCC), Punta Arenas: Río de las Minas (Gordon 1994). (Fig. 3:12).

Orynipus kuscheli Hofmann, 1972 (Fig. 1:13)

De forma elíptica, alargada y semiconvexa. Con todo el cuerpo de color anaranjado amarillento o castaño oscuro. Pilosidad semiabundante de color amarillenta, sobre todo en los élitros. El bordes delantero del pronoto se proyecta por sobre la cabeza.

Esta especie se distribuye en Chile desde la región del Bío-bío hasta la región de Magallanes 
y Antártica Chilena (González 2010²). Las localidades encontradas tanto en la literatura como en las colecciones son: Provincia de Magallanes: Isla Riesco: Punta Rocallosa (UCCC), Reserva Forestal Magallanes (CZIP), Punta Arenas (Gordon 1994). Comuna Cabo de Hornos y Antártica Chilena: Puerto Navarino (UCCC). (Fig. 3:13).

\section{Orynipus ultimensis Gordon, 1994 (Fig. 1:14)}

Especie muy similar externamente a la anterior, la única diferencia que se puede encontrar con la otra especie es que O. ultimensis posee una franja de pilosidad densa, franja que parte desde el $1 / 3$ de la zona basal hasta el 1/3 de la zona apical (Gordon 1994).

Esta especie es sólo conocida para la región de Magallanes y Antártica Chilena (González $2010^{2}$ ). No se encontró material de esta especie en las colecciones revisadas y sólo contamos con la localidad citada en la literatura: Provincia de Última Esperanza: Fiordo Exmouth: Glaciar Pio XI, Estero Worsley, Isla Guarello (Gordon 1994). (Fig. 3:14).

Stictospilus darwini Brèthes, 1925 (Fig. 1:15)

Cuerpo de forma elíptica que va se agudiza hacia la parte posterior, anteriormente truncado $y$ plano dorsoventralmente. Cuerpo generalmente de color marrón rojizo, pudiendo ser la cabeza y el pronoto más oscuro, casi negro. Pilosidad corta semi abundante y desordenadamente distribuida en el pronoto. Élitros con poca pilosidad, larga y separada equidistantemente, distribuida en línea que van desde la base hasta el ápice. La línea pilosa que está más cerca de la sutura elitral con pelos más densos y cortos.

Esta especie se distribuye en Chile desde la región de Valparaíso hasta la región de Magallanes y Antártica Chilena (González 2010²). No se encontraron ejemplares colectados en Magallanes de esta especie en las colecciones visitadas y tan sólo tenemos la localidad citada en la literatura. La localidad citada es: Ultima Esperanza: Puerto Natales: Cerro Dorotea (Alfredo Lüer com. pers.). Patagonia: Valle del Lago Blanco (Brèthes 1925b), es probable que se refiera a la localidad situada en la porción chilena de la isla Tierra del Fuego. (Fig. 3:15).
Parasidis australis (Gordon, 1977)

Gordon (1977) describió Sarapidus australis, nuevo género y especie procedente de la Región de Magallanes y Antártica Chilena que posteriormente González (2008) sinonimizó el género Sarapidus con Parasidis. Según Honour \& González (2010), $P$. australis es una especie muy cercana a Parasidis brethesi, esto se desprende de la descripción y los dibujos de Gordon (1977) y que morfológicamente deben ser muy parecidas.

Esta especie es sólo conocida para la región de Magallanes y Antártica Chilena (Gordon 1977). No se encontró material de esta especie en ninguna colección revisada y tan sólo contamos con la localidad citada en la literatura. Provincia de Magallanes: Punta Arenas (Gordon 1977). (Fig. 3:18).

\section{Neorhizobius fuegensis (Brèthes, 1925)}

Esta especie es muy parecida a N. sanguinolentus, pero $N$. fueguensis sólo posee una gran mancha de color sangre en cada élitro. Esta mancha se ubica en la base elitral y no alcanza la zona media, y lateralmente ocupa el tercio central del élitro. (Brèthes 1925b).

Especie sólo conocida para la región de Magallanes y Antártica Chilena (Brèthes 1925b). No se encontró material de esta especie en ninguna colección revisada y tan sólo contamos con la localidad citada en la literatura.

Provincia de Tierra del Fuego: Bahía Inútil. (Brèthes 1925 b). (Fig. 3:17).

Neoryssomus germaini (Crotch, 1874) (Fig. 1:16)

Esta especie posee una forma semicuadrada y plana dorsoventralmente. El cuerpo es completamente negro. Pronoto ancho, plano en la base y formando un arco hacia adelante, sobrepasando y tapando la cabeza. Borde lateral y anterior del pronoto de color amarillo pálido mientras que el disco es de color negro y cubierto de pilosidad corta e irregular. Élitros completamente negros, con puntuaciones finas y con pilosidad algo dispersa y desordenada.

El ejemplar de $N$. germaini, presenta algunas diferencias en la puntuación elitral con respecto a las poblaciones de más al norte, por lo que probablemente podría tratarse de otra especie o sólo 
una variación morfológica de ésta. Esto se debe confirmar con la ayuda de más ejemplares ya que sólo se cuenta con un uno.

Especie que se distribuye desde la región de Valparaíso hasta la región del Biobío (González 2010²). Localidad de colecta: Provincia de Magallanes: Río San Pedro, Península de Brunswick (bajo corteza de canelo) (CPAZ). (Fig. 3:16).

\section{DISCUSIÓN}

De un total de 18 especies de chinitas registradas en este trabajo para Magallanes, las especies $N$. germaini, S. peregrina, A. deficiens, A. angulifera y $A$. bipunctata constituyen nuevos registros para la región. S. peregrina, $A$. deficiens y $A$. angulifera tenían registros previos en regiones chilenas cercanas a Magallanes, y habían sido citadas para la región de Aisén (González 2010²) y en el caso particular de S. peregrina se encontraba citada en la localidad argentina de Río Turbio (Gordon 1994), localidad que es colindante y muy cercana a Puerto Natales. Por otra parte, $N$. germaini amplía notablemente el rango de distribución del género y de la especie, ya que Neorysommus había sido citado hasta la región de los Lagos, mientras que la especie había sido citada hasta la región del Biobío en Chile (González 2010²).

Por otra parte, las especies $P$. australis, $N$. fuegensis y $O$. ultimensis serían las únicas especies endémicas de la región, mientras que $C$. patagonica sería una especie endémica del extremo austral de Sudamérica, encontrándose en Tierra del Fuego de Chile y Argentina e isla Navarino. El resto de las especies poseen una distribución más amplia, tanto dentro de Chile como en Argentina. Adicionalmente, las especies $H$. convergens y $A$. bipunctata son dos especies aloctonas y abundantes en la mayor parte de Chile. Estas especies fueron introducidas al país, por ser buenos controladores biológicos como depredadores de áfidos (Aguilera et al. 1981, González 2006). H. convergens fue introducida a principios del siglo XX sin éxito y luego en 1961, empezando a registrarse en 1967 en Arica y luego en todo el país (Aguilera et al. 1981). Por otra parte, A. bipunctata fue introducida en 1941 y se ha vuelto muy común actualmente (Zúñiga 1985). Los registros de estas especies introducidas en Magallanes son apenas de unos pocos ejemplares y han sido colectados en áreas con fuerte impacto ganadero y donde por este medio probablemente pudo haber sido introducido. Sin embargo, al parecer no lograron naturalizarse con éxito en la región debido a que no han sido nuevamente recolectadas en la región por más de 30 años en el caso de $H$. convergens y 50 años en el caso de A. bipunctata.

Por otra parte, aún existen algunas especies conflictivas en los registros y se duda de la validez taxonómica. Tal es el caso de P. australis, donde no se ha esclarecido si realmente es una especie independiente ya que no se ha podido diferenciar esta especie de otras con distribución más boreal, debido a la pobre descripción original y la escasez de antecedentes y nuevas recolectas en la localidad tipo (Honour \& González 2010). N. fuegensis no se ha vuelto a recolectar desde su descripción, lo que ha permitido sospechar que podría ser sinónimo de Neorhizobius sanguinolentus, lo cual no se ha podido corroborar ya que el holotipo se encuentra perdido (González 20061). Un caso parecido fue la presencia de E. eschscholtzi en la región. Hoffman (1970) citó a esta especie para Magallanes, pero dicha cita se habría tratado de un error de identificación de autor, al haber confundido a E. eschscholtzi con algunos ejemplares de E. magellanica. Sin embargo, recientemente esta especie fue recolectada en Última Esperanza y fue reincorporada como parte de la entomofauna regional (González 2008).

Según Pisano (1977) y Dollenz (1995) el paisaje fitogeográfico de esta región es modelado por un gradiente climático longitudinal, el cual comienza en la vertiente occidental de la cordillera con una zona de alta humedad, dando origen a un bosque lluvioso siempreverde y luego a una zona de transición ecotonal de bosque-estepa, con humedad intermedia. Esta da origen a los bosques caducifolios y matorrales, y por último culmina en una zona árida, correspondiente a la estepa patagónica en la vertiente oriental de la cordillera de los Andes. Según los resultados de este trabajo, desde un punto de vista biogeográfico, las especies de coccinélidos muestran una patrón de distribución que respondería a los cambios del paisaje en la región, siguiendo las estructuras fitogeográficas propuestas para la región de Magallanes. Así en la estepa patagónica podemos encontrar la mayor parte de las especies nativas como es el caso de: E. magellanica, E. eschscholtzi, C. limbicollis, C. germanii, C. patagónica y probablemente $N$. fuegensis. A estas especies se les puede encontrar 
en la estepa y más comúnmente bajo el follaje de plantas rastreras (e.g Senecio patagonicus Hook et Arn). Sin embargo, estas especies pueden encontrarse también en zonas de transición estepa-bosque (e.g C. patagónica) e incluso, en jardines y parques de ciudades (e.g. E. magellanica y C. germanii). Las especies de los géneros Eriopis y Cycloneda prefieren los estratos herbáceos y son muy abundantes en este estrato, pero también pueden ocupar en menor grado los estratos arbustivos y arbóreos tanto de las especies vegetales nativas, como de especies introducidas (Rebolledo et al. 2007), lo que sugiere una elasticidad en la selección de hábitat por parte de estos géneros.

Por otra parte, en la zona de transición bosque-estepa, en donde predominan los bosquetes de Nirre-Lenga (Fagaceae) y donde además existen arbustos de denso follaje, se pueden encontrar las chinitas nativas $A$. angulifera, $A$. deficiens, $N$. sicardi, S. nigrodorsata, S. peregrina O. kuscheli, y probablemente $S$. darwini y $P$. australis. Todos los géneros mencionados han sido colectados mayoritariamente sobre vegetación nativa arbustiva y/o arbórea (e.g. A. angulifera) por lo que deben tener hábitos principalmente arborícolas, ocupando mayoritariamente los estratos arbustivos y arbóreos que ofrece la zona de transición.

Por último, en las zonas más húmedas de la región, como los bosques siempreverdes nor $y$ sur-patagónicos, se encuentran como habitantes exclusivos de estos ambientes las especies $O$. ultimensis por el norte y N. germanii por el sur. Sin embargo, también podemos encontrar a las especies $O$. kuscheli y $A$. angulifera en los bosques del extremo sur como los de Isla Navarino y Cabo de Hornos. Todos estos géneros han sido capturados en asociación con bosque de Nothofagus en latitudes más septentrionales (González 20061). Un ejemplo es $O$. ultimensis que ha sido capturado en el follaje de Nothofagus betuloides, mientras que $N$. germaini tiene actividad nocturna sobre los troncos de Nothofagus obliqua (Gonzalez 20061). Sin embargo, esta especie al parecer puede estar asociado a troncos de otros árboles que habitan en los bosques de Nothofagus como es el caso de Drimys winteri.

Hasta ahora la información referente a las chinitas de Magallanes es aún incompleta. Se hace necesario completar la información con más colectas de la mayoría de los coccinélidos, para esclarecer los estatus taxonómicos de algunas especies, así como también, complementar la información con aspectos biológicos y datos de distribución en la región. Sin embargo, trabajos recopilatorios como este son necesarios para conocer el estado actual de la diversidad biológica de una región. Nos permite conocer grosso modo la posible amenaza de especies introducidas, así como también permite comprender mejor como se está estructurando esta biodiversidad en los sistemas patagónicos australes, información que puede ser usada para tomar futuras medidas de protección y conservación de la biodiversidad austral.

\section{AGRADECIMIENTOS}

El autor agradece sinceramente a Guillermo González, por sus aportes literarios, registros y en la identificación de las especies. A Ricardo Honour y Alfredo Lüer, por facilitar los registros de Magallanes de su colección. A Vicente Pérez, Elvira Solar, Patricia Estrada y a Mario Elgueta, por permitir revisar las colecciones del CZIP, UCCC, UMCE y MNHN, respectivamente. A la Licenciada Gloria Gallegos por las fotografías enviadas de UCCC y por los comentarios del texto. $Y$ por último a Eduardo Faúndez por enviar una foto in situ de E. magellanica.

\section{LITERATURA CITADA}

Aguilera, A. 1987. Nuevas localidades para Stethorus histrio Chazeau (Coleoptera: Coccinellidae) en Chile. Revista Chilena de Entomología 15:33-36.

Aguilera, A., H. Vásquez \& N. Hichins 1981. Distribución geográfica de Hippodamia convergens Guérin en Chile (Coleoptera: Coccinellidae). Simiente 51:3-4.

Aguilera, A. \& S. Rojas 1990. Scymnus (P.) loewii Mulsant, nuevo coccinélido (Coleoptera: Coccinellidae) para Chile. Revista Chilena de Entomología 18: 93-94.

Arias, E. 2000. Coleópteros de Chile. Fototeknika. Santiago de Chile. 209 pp.

Brèthes, J. 1925 a. Sur une collection de Coccinellides (et un Phalacridae) du British Museum. Anales del Museo Argentino de Ciencias Naturales 33:145-175. 
Brèthes, J. 1925 b. Coleóptères, principalement Coccinellides, du British Museum. Nunquam Otiosus 3: 16.

Dollenz, O. 1995. Los árboles y bosques de Magallanes. Ediciones de la Universidad de Magallanes. Punta Arenas, Chile. 123 pp.

Elgueta, M. \& G. Arriagada 1989. Estado actual del conocimiento de los coleópteros de Chile (Insecta, Coleoptera). Revista Chilena de Entomología 17:5-60.

González, G. 2008. Lista y distribución geográfica de Coccinellidae (Insecta: Coleoptera) presentes en Chile. Boletín del Museo Nacional de Historia Natural 57:77-107.

González, G. \& N. Vandenberg 2006. Review of lady beetles in the Cycloneda germainii species complex (Coleoptera; Coccinellidae: Coccinellinae: Coccinellini) with descriptions of new and unusual species from Chile and surrounding countries. Zootaxa 1311:13-50.

Gordon, R. 1977. Classification and phylogeny of the new world Sticholotidinae (Coccinellidae). Coleopterist Bulletin 31:185-228.

Gordon, R. 1994. South American Coccinellidae (Coleoptera). Part IV: definition of Exoplectrinae Crotch, Azynae Mulsant, and Coccidulinae Crotch; a taxonomic revision of Coccidulini. Revista Brasileira de Entomologia 38:681-775.

Hofmann, W. 1970. Die Gattung Eriopis Mulsant (Col. Coccinellidae).Mitteilunger der Muncher Entomologischen Gesellschaft 60:102-116
Honour, R. \& G. González 2010. Revisión del género Parasidis Brèthes (Coleoptera, Coccinellidae, Microweiseini). Boletín de la Sociedad Entomológica Aragonesa 46: 59-70.

Pisano, E. 1977. Fitogeografía de Fuego-Patagonia chilena. I. Comunidades vegetales entre las latitudes 51 y $56^{\circ}$ Sur. Anales Instituto $\mathrm{Pa}$ tagonia (Chile) 8: 121-250.

Rebolledo, R., R. Palma, C. Klein \& A. Aguilera 2007. Coccinellini (Col. Coccinellidae) presentes en diferentes estratos vegetacionales en la IX región de la Araucanía (Chile). Idesia 25:63-71.

Zabala, J., J. Iturralde \& M. Saloña 2003. Etnoentomología de la Vaquita de San Antón o Mariquita (Coccinella septem-punctata) en el País Vasco (Coleoptera: Coccinellidae). Boletín de la Sociedad Entomológica Aragonesa 33:253-269.

Zúñiga, E. 1967. Lista preliminar de áfidos que atacan cultivos en Chile, sus huéspedes y enemigos naturales. Agricultura Técnica 27:165-177.

Zúñiga, E. 1985. Ochenta años de control biológico en Chile. Revisión histórica y evaluación de los proyectos desarrollados (1902-1983). Agricultura Técnica 45: 175-183.

Zúñiga, E., R. Van Den Bosch, J. Pnea, F. Gruber 1986. Control biológico de los áfidos (Hom.: Aphididae) de los cereales en Chile II. Obtención, introducción y cuarentena de depredadores y parasitoides. Agricultura Técnica 46:479-487. 
A. ZÚÑIGA 